the JAMA benchmarks nor included the HON seal included only 2 improper treatment measures.

This study highlights the variety of misinformation available on the internet regarding prehospital care of snake envenomation and the unreliability of the HON seal and JAMA benchmarks as markers of accuracy. As the internet becomes an increasing source of medical information for both the public and clinicians, the importance of accurate websites becomes imperative. Clinicians and patients should be aware of the high variability of internet information regarding snake bite prehospital care.

Sarah J. Barker, MD

Nathan P. Charlton, MD

Christopher P. Holstege, MD

Charlottesville, VA, USA

\section{Do Waterborne Coliform Bacteria Increase in Sierra Nevada Mountain Wilderness Area Lakes and Streams During Drought Periods?}

Objectives.-Humans may acquire waterborne infections in remote wilderness environments. We hypothesize the risk of waterborne infection increases during drought years.

Methods.-Water was collected from wilderness area lakes and streams in the Sierra Nevada Mountains of California during summer 2007 and 2008, and results compared to a previously published data set from this laboratory. Precipitation during water years 2007 and 2008 was $45 \%$ and 55\% of normal, respectively. Collection locations matched those from prior years in Yosemite, Kings Canyon-Sequoia (SEKI) National Parks and Forest Service Wilderness. Land use was categorized as predominate impact from either: 1) wildlife (W), 2) day-hike (DH), 3) backpackers (BP) only, or 4) pack animals (PA). Water was collected in sterile tubes and Millipore coliform samplers and analyzed at the University of California, Davis research laboratory, where bacteria were harvested, then subjected to analysis using standardized techniques. Coliforms were used as a marker of infection risk; samples showing $>100$ CFU/100 mL were considered positive. Statistical analysis to compare among site categories was performed using Fisher exact test.

Results.-A total of 168 sites were sampled (84 each year) among the 3 types of wilderness. Prevalence of coliforms is listed by area and land use (\% positive, raw number positive sites/total sites):

\begin{tabular}{llccc}
\hline & Wild & Day-hike & Backpack & Pack animal \\
Location & $(W)$ & $(D H)$ & $(B P)$ & $(P A)$ \\
\hline
\end{tabular}

Yosemite $\quad 35 \%(5 / 13) \quad 10 \%(2 / 10) \quad 20 \%(5 / 23) \quad 78 \%(18 / 23)$

$\begin{array}{lllll}\text { SEKI } & 0 \%(0 / 14) & 14 \%(1 / 7) & 17 \%(2 / 12) & 68 \%(23 / 34)\end{array}$

Forest wild $20 \%(1 / 5) \quad 50 \%(1 / 2) \quad 17 \%(1 / 6) \quad 95 \%(18 / 19)$

$\begin{array}{lllll}\text { Totals } & 19 \%(6 / 32) & 21 \%(4 / 19) & 20 \%(8 / 41) & 75 \%(59 / 76)\end{array}$

No difference was found comparing 2007 to 2008; coliform prevalence was significantly higher in PA use areas when compared to either $\mathrm{W}, \mathrm{DH}$, or $\mathrm{BP}$ areas $(\mathrm{P} \leq .05)$. Results were also compared to coliform prevalence from 364 samplings collected during 5 nondrought years (2002-2006) previously reported as follows: W 9\%, DH 12\%, BP 18\%, and PA $63 \%$. Coliform prevalence was higher in the drought years compared to nondrought, in $\mathrm{W}, \mathrm{DH}$, and PA areas but only significant in PA areas $(59 / 76$ vs $70 / 111 ; P<.05)$.

Conclusion.-During drought years, wilderness water coliform prevalence was significantly increased compared to nondrought years in those areas used by pack animals.

Robert W. Derlet, MD

John R. Richards, MD

Sacramento, CA, USA

Kemal A. Ger, PhD

Davis, CA, USA

\section{A Novel Technology for Assessing Cerebral Blood Flow at Altitude}

Introduction.-Most current assessments of cerebral hemodynamics at altitude rely on transcranial dopplers (TCDs). This technology does not monitor cerebral blood flow (CBF), per se, but rather blood flow velocity. Equating CBF to blood flow velocity is predicated on multiple assumptions that remain unproven at altitude, such as constant diameter of mediumsized arteries. An increase in middle cerebral artery velocity as measured by TCD is interpreted as evidence of vasospasm within the context of subarachnoid hemorrhage, for example, but vasodilation within the context of ascent to altitude. Current techniques to directly assess CBF, such as Xenon CT (XeCT), are either too cumbersome or too expensive to allow serial measurements to assess the temporal dynamics of changes in CBF during ascent to high altitude.

We present a human feasibility study of a technique, previously validated in animal models, that allows measurement of $\mathrm{CBF}$ that is uniquely suited to investigation of blood flow changes at altitude.

Methods.-Near infrared spectroscopy coupled with intravenous injection of indocyanin green dye (ICG-NIRS) was used to measure a CBF index in 3 patients undergoing laparoscopic surgery to assess feasibility of this technique in humans.

Results.-Two of the 3 patients exhibited an increase in $\mathrm{CBF}$ index of $40 \%$ and $59 \%$, during insufflation of $\mathrm{CO}_{2}$. The third patient exhibited a paradoxical decrease in $\mathrm{CBF}$ index of $39 \%$.

Conclusions.-The combination of ICG-NIRS and determination of CBF index is a promising, minimally invasive, portable and straightforward technique to provide repeated quantitative information regarding changes in CBF. This method is well suited to use in the alpine environment. Further refinements in technique and equipment, as well as formal validation studies in humans, are needed to increase the utility of ICGNIRS.

Matthew R. Sanborn, MD W. Andrew Kofke, MD Philadelphia, PA, USA 